\title{
Factors Related to Nurses' Attitudes Towards the Suicidal Patient: An Integrative Review
}

\author{
Karen Patterson Stevens ${ }^{1}$ and Mary A Nies ${ }^{2 *}$ \\ ${ }^{1}$ Jonas Scholar, Idaho State University, Ketchum, Idaho, USA \\ ${ }^{2}$ Director of Nursing Research, Joint Appointment MPH Program, Division of Health Sciences, Idaho State University, Pocatello, Idah, USA
}

\begin{abstract}
Aims and objectives: To conduct an integrative review of the literature to describe what is known about the factors related to nurses' attitudes towards the care of the suicidal patient.

Background: Suicide, a preventable public health problem, is at epidemic levels in the United States and globally. Regardless of their specialty or their hospital setting, nurses will encounter and provide care for suicidal patients. Little is known about the factors that collectively affect nurses' attitudes towards caring for the suicidal patient in general hospital settings.

Design: An integrative literature review.

Methods: To better understand factors that influence nurses' attitudes towards the care of the suicidal patient, a literature search was conducted to identify relevant articles written in English between 2000 and 2017. This search was conducted in eight online databases using relevant keywords and including only scholarly, peerreviewed studies. A narrative approach was used to analyze the data.

Results: After reviewing titles and abstracts, a final yield of 23 studies were included in this review. The literature described various factors that affect nurses' attitudes towards suicide or suicidal patients. The evidence suggests that the relationships are significant and complex. Nurses' attitudes are affected by components of attitude as well as by socio-demographic background and self-reported professional capacity to care for this vulnerable population.

Conclusions: The attitude a nurse has towards the care of a suicidal patient is affected by internal and external factors. Knowledge and self-reported professional capacity to care for the suicidal patient are significant factors affecting the nurses' attitude. More information is needed to understand the relationship between compassion satisfaction and compassion fatigue and attitude.
\end{abstract}

Relevance to clinical practice: Targeted education that prepares nurses for interactions with suicidal patients is needed across all hospital settings. This education should be informative, explanatory, and challenging to personal beliefs and mindsets.

\section{Introduction}

Suicide, a preventable public health problem, is at epidemic levels in the United States (US) and [1]. Suicide accounts for more global deaths than homicide, motor vehicle collisions, and war [2]. More than 800,000 suicides occur each year globally [3] and suicide is the second leading cause of death in the 15-29-year cohort [4]. In the US, the National Survey on Drug Use and Health (NSDUH) found that in 2014, 9.4 million adults in the US had suicidal thoughts, 2.7 million adults made a suicide plan, and 1.1 million adults made an actual attempt to end their life [5]. Of those 1.1 million adults who attempted suicide, $55.2 \%$ sought medical attention and $42.7 \%$ stayed in a hospital for at least one night following the attempt [5]. The average length of stay for patients admitted to the hospital for suicide thoughts and behaviors is 5.6 days [6]. Additionally, the Agency for Healthcare Research and Quality (AHRQ) found that in 2013, 1\% of all emergency visits were related to suicide ideation [7].

The intersection of suicide, suicidal ideation, and suicide attempts within the healthcare system is significant and due to this, nurses routinely interact with patients experiencing suicidal thoughts and behaviors. Detecting and treating suicidal patients in all nurse settings is a national nursing priority [8] and the 2012 National Strategy for Suicide Prevention stresses transforming the health care systems to significantly and proactively reduce suicide [9]. Many studies have shown that a majority of people who complete suicides have had recent contact with health care professionals before they die [10]. In fact, suicide is the second leading cause of hospital sentinel events [11]. Literature shows that nurses have the best opportunity to identify and intervene with potentially suicidal patients [1] and nurses who care for suicidal patients have the ability to provide positive therapeutic care and to potentially intercede with life-saving measures [1].

Negative attitudes among nursing personnel present a major obstacle in providing appropriate and timely interventional care for the

Correspondence to: Karen Patterson Stevens, Jonas Scholar, Idaho State University, Ketchum, Idaho, USA, Tel: 208-309-5162; E-mail: karenpstevens@ gmail.com

Key words: suicide, suicidal patient, nurse, attitude, nurses' attitudes, professional capacity, compassion, compassion satisfaction, compassion fatigue

Received: February 07, 2018; Accepted: February 21, 2018; Published: February 26,2018 
suicidal patient [12]. Evidence suggests that negative attitudes towards suicidal patients may adversely impact patient outcomes [11]. Negative attitudes may reinforce a patient's feeling of perceived burdensomeness [13] and this could lead to an exacerbation of hopelessness.

A nurse's positive attitude toward a suicidal patient may be key in preventing a future suicide attempt or a completed suicide [14]. The attitude a nurse has towards a suicidal patient has the potential to have a direct impact on patient safety, patient quality of care, and patient outcomes [1].

\section{Aims}

The purpose of this integrative review is to critically review and analyze current and seminal research related to nurses' attitudes towards the care of the suicidal patient. This review will explain the components of attitude and will explore multidimensional factors that influence and predict nurses' attitudes towards the suicidal patient. Among those factors will be the components of attitude, the socioeconomic demographic background of nurses, nurses' selfreported level of professional capacity to care for the suicidal patient, and nurses' level of compassion satisfaction and compassion fatigue, burn-out, and secondary traumatic stress.

\section{Methods}

To better understand factors that influence nurses' attitudes towards the care of the suicidal patient, a variety of resources were used to search the current literature. The following databases were searched: EBSCO, Google Scholar, PubMed, Cochran Library, CINAHL Complete, Clinical Key, ResearchGate, and ProQuest. Queries were made across nursing, healthcare, and psychology disciplines. Key search terms were identified as nurses, suicide, attempted suicide, attitudes, nurses' attitudes, compassion, compassion fatigue, nurse burnout, secondary traumatic stress, and components of attitude. An initial unrestricted search provided 12,253 articles.

Narrowing the search strategy to scholarly peer-reviewed articles published between 2000 and 2017 yielded 289 articles. The title of these articles and their abstracts were reviewed for additional inclusion criteria including quantitative, mixed-methods, and qualitative studies that specifically researched nurses' attitudes towards the direct care of the suicidal patient. This resulted in 47 studies. After eliminating duplicate studies and non-English language studies, the final yield was 23 studies. Most of these studies $(N=20)$ were international. They included Greece $(n=2)$, Sweden $(n=3)$, Spain $(n=1)$, Brazil $(n=$ $2)$, Australia $(n=3)$, India $(n=1)$, Taiwan $(n=2)$, Turkey $(n=1)$, Netherlands $(n=1)$, United Kingdom $(n=1)$, Finland $(n=1)$, and Japan $(n=2)$. Two current studies and one seminal study $(n=3)$ took place in the United States.

Only two of the final 23 studies used a sample of all nurses working in a general hospital setting. The other studies used a sample of emergency department (ED) nurses $(n=3)$, mental health nurses $(n=$ $2), \mathrm{ED}$ and mental health nurses $(n=2)$, nurses combined with various other clinical staff such as physicians, certified nursing assistants, mental health assistants $(n=7)$, non-clinical hospital personnel $(n=1)$, other clinical personnel such as physicians and mental health assistants $(n=3)$, students $(n=1)$, urban residents $(n=1)$, and patients $(n=1)$.

The final yield of studies $(N=23)$ mostly focused on attitudes towards suicide or suicidal patients, however, several studies were retained that studied nurses' attitudes towards mental health patients and nurses' attitudes towards patients who self-harm. Most of the studies were quantitative studies, one was a mixed-methods approach, and one was a qualitative study.

\section{Results}

Nurses interact with patients having suicidal thoughts and behaviors across all hospital settings. Because of the significant amount of time nurses spend interacting with patients, they are considered front-line in identifying, managing, and intervening in the care of patients with suicidal thoughts and behaviors [15]. Regardless of their specialty area, nurses will encounter and provide care for suicidal patients [1].

What is attitude? Attitude is conceptualized as having three interdependent components: the affective component, the cognitive component, and the behavioral component [16]. The affective component is covert and consists of an emotional response to an issue or a person [16]. The cognitive component of attitude is also covert and refers to the thoughts and the beliefs an individual has towards an issue or a person [17]. The behavioral component is overt, and it refers to the observable responses and actions towards an issue or person [18]. This model of attitude and these three factors are important in understanding the overall concept of attitude as it relates to nurses' attitudes towards the suicidal patient.

Many attempts have been made to describe the factors that quantitatively measure attitudes towards suicidal patients. Ouzouni, et al. [16] headed a study to develop a survey tool designed to quantify and better understand health care personnel's attitudes towards the suicidal patient. In the development of this measurement instrument - the Attitudes Towards Attempted Suicide Questionnaire (ATAS-Q) - previously validated attitudinal measures from the Suicide Opinion Questionnaire [19] were collected and modified for use with a pilot study of doctors and nurses working in general hospital settings. A second study with a sample of 186 doctors and nurses found that eight factors accounted for $55.451 \%$ of the total variance [16]. According to Ouzouni \& Nakakis [16], each of these eight factors represents a different dimension that explains health care personnel's attitudes towards the suicidal patient. These eight factors can be further organized into the three components of attitude: cognitive, affective, and behavioral [16].

Cognitive component: The cognitive component of attitude refers to what a person knows and what a person believes about an issue or about another person. Four factors fall under the cognitive component of attitude: Positiveness, Religiosity, Personality traits, and Mental illness [16]. According to Ouzouni \& Nakakis [16] Positiveness measures both positive and negative attitudes of health care personnel towards attempted suicide patients. Religiosity considers suicide and attempted suicide and its relationship to religious issues. Personality Traits refers to the character and traits of those people who attempt suicide. Finally, Mental Illness refers to associating suicide and suicide attempts with mental illness [16].

Affective component: The affective component of attitude refers to what a person feels about another person or object. Acceptability is the factor designed to measure for the affective component of attitude [16]. Acceptability refers to the level of acceptance for suicide and suicide thoughts and behaviors that nurses have towards patients [16].

Behavioral component: The behavioral component of attitude refers to responses and actions towards an issue or person. Three factors - Professional role and care, Manipulation, and Discrimination - were designed to measure the behavioral component of attitude [16]. Professional role and care refers to the care that attempted suicide patients should receive and aspects of the professional role and work 
environment of the nurses [16]. Manipulation refers to the belief amongst nurses that attempted suicide patients try to manipulate their environment. Finally, Discrimination refers to discriminatory attitudes of nurses towards the suicidal patient [16].

Another attempt to quantify attitude was developed by Botega, et al. [20]. In this 21 item questionnaire, Botega [20] uses three subscales to measure attitude: Feelings towards the patient, Professional capacity, and Right to suicide. In the development of this tool, 317 nurses and nursing assistants were placed in three separate focus groups to produce a list of 54 propositions on suicidal behavior [20]. After a factor analysis using maximum likelihood and orthogonal Varimax rotation, 25 of the highest scoring propositions were used to form the Suicide Behavior Attitude Questionnaire (SBAQ).

Demographics and attitude: Socio-demographics such as age, gender, education, experience, and nursing specialty are some factors that have been explored when studying overall attitude of nurses towards the care of the suicidal patient. A study by Neville \& Roan [11] sought to investigate nurses' attitudes towards suicidal patients in the medical/surgical area of a general hospital. A convenience sample of 45 nurses was used in this descriptive, non-experimental study design. Demographic data was collected, and this included gender, nursing specialty, age, education, religion, ethnicity, and race. The nurses' overall attitude was quantified using ATAS-Q [16].

Correlational analyses were performed on the demographic variables and the ATAS-Q items. The possible overall score on the ATAS-Q ranges from 80 to 400 with the higher score indicating a more positive attitude towards suicidal patients [16]. The study showed a mean score of $262.51(S D=16.34)$ and the scores on the ATAS-Q were not correlated to the categories of the demographic variables [11]. However, there was a significant inverse relationship with Positiveness and religion showing that those nurses who identified with religion had more positive attitudes towards suicidal patients. Nurses' age also showed a significant relationship to Positiveness with younger nurses having a higher positive attitude than their older colleagues [11].

Another study examined demographics and nurses' attitudes towards the suicidal patient using the SBAQ survey instrument. In this study, nurses and nursing assistants $(n=317)$ were given the SBAQ survey to determine the associations between characteristics of the nursing staff and their attitudes towards suicidal patients [20]. The results of this study indicated that older nursing staff ( $>50$ years of age) compared to younger (20-29 years of age) showed a more condemnatory attitude towards suicidal patients based on a comparison of means. In addition, the nursing staff who attended church on a regular basis had a more condemnatory attitude towards suicidal patients than those who did not attend church [20].

In a study in Turkey, a group of physicians $(n=158)$ and a group of nurses $(n=206)$ were given a questionnaire to study the predictors of therapeutic versus non-therapeutic reactions to suicide attempters [20]. The findings indicated men scored much higher on average than women on suicide as a sign of mental illness. On the whole, the study showed that women were more tolerant and sympathetic than men towards the care of the suicidal patient [10].

A 2005 study in Taiwan investigated the attitudes of ED nurses $(n=155)$ to patients who attempted suicide. This study showed the significance of nursing education on nurses' attitudes towards the suicidal patient. Results showed that there was a significant relationship between nurses' level of education and their attitude towards suicidal patients [21]. The higher the level of education, the more positive the nurses' attitude was towards the suicidal patient [21]. Additionally, as a previous described study showed [20]. ED nurses who indicated they had no association with religion had more positive attitudes towards suicidal patients [21].

Professional capacity and attitude: The SBAQ includes a subscale that measures the perceived professional capacity of the nurse to care for the suicidal patient. A higher score on this subscale indicates the professional capacity of the nurse to care for and work with patients at risk for suicide. Professional capacity refers to the ability of the nurse to care for the suicidal patient and includes concepts such as professional skills, perception, security, and capability [20].

According to the American Nurses Association (ANA), the professional role competency of a nurse is a responsibility shared by the individual professional nurse and by the nursing profession [22]. Nurses' professional capacity refers to the nurses' understanding of what the professional expectations are of a nurse, as well as the nurses' preparation to meet those expectations [22]. Botega [20] found that nurses with a higher self-reported level of professional capacity were caregivers who had less of a condemnatory attitude towards suicidal patients. A more condemnatory attitude was higher among the staff who had never handled suicidal patients than those who were experienced with this type of nursing care $(M=404.5$ and 382.4, $p=$ 0.08 ) indicating a more positive attitude towards suicidal patients was correlated with the perception of the ability to care for these patients [20].

A study in Australia (2002) was undertaken in order to develop a survey tool to measure ED nurses' attitudes towards patients who selfharm. The results of the study showed that confidence in the ability to assess and refer self-harm patients was correlated to positive attitudes towards those patients [23]. The authors concluded that if staff perceive themselves as skilled, they are less likely to demonstrate negative attitudes to the self-harm patient [23].

A Florida State University (FSU) study used the Professional capacity subscale from the SBAQ to assess mental health professionals' attitudes towards the suicidal patient. The 2012 repeated measure design study at FSU showed significant improvement in nurses' attitudes between Time 1 and Time 2 after the nurses completed a training program offered by the American Association of Suicidology [24]. The authors concluded that when clinicians perceive themselves as prepared and confident to care for the suicidal patient and to respond to potential suicide risk, their attitudes towards the suicidal patient will significantly improve [24].

An additional study demonstrated the importance of education and preparation in improving attitudes towards the suicidal patient. A 2014 study in Sweden sought to focus on stigmatization towards and discrimination against suicidal patients by nursing staff [12]. This study revealed that mental health nurses have more positive attitudes towards patients if their knowledge about mental illness is less stigmatized. The authors concluded that educational programs that improve knowledge through challenges to personal beliefs and mindsets lead to improved overall attitude towards the care of the suicidal patient [12].

A quasi-experimental study in Taiwan studied a group of secondyear nursing students $(n=174)$. The students were divided into two groups: the intervention group $(n=95)$ and the control group $(n=$ 79). The intervention group attended a four-hour suicide education program designed to educate the students on the theoretical background of suicide and to inform the students of various intrapsychic conflicts 
that lead to suicidal thoughts [25]. Data analysis showed there was no significant differences in the group before the educational intervention. After the educational intervention, the intervention group was less judgmental in their morality towards suicidal patients and showed a belief that those who attempt suicide are expressing psychic pain and reaching out for help [25]. The authors concluded that the synthesis of knowledge regarding theoretical perspectives on suicide led the experimental group to become less judgmental and to experience improved attitudes towards caring for the suicidal patient [25].

A study by Berlim [15] demonstrated the importance of education in improving the professional capacity of those caring for the suicidal patient. The study evaluated clinical $(n=102)$ and non-clinical $(n=40)$ staff after a 3-hour training video. The SBAQ was used to survey the sample using a pre-test, post-test design. As the previous studies have shown, the attitudes of both clinical and non-clinical staff improved after the intervention [15]. The authors concluded that "the need for enhanced training on understanding, assessing, and preventing suicidal behavior" is necessary for all hospital personnel [15].

Compassion and attitude: A survey tool that is used to assess compassion satisfaction and compassion fatigue in nurses is known as the Professional Quality of Life Scale (ProQoL). This survey was developed to evaluate how both the positive and negative aspects of helping others influences a professional's overall quality of life [26]. According to the survey author, the professional quality of life is affected by two aspects: the positive or compassion satisfaction and the negative or compassion fatigue. Compassion fatigue is further broken down into two additional components: burn-out and secondary traumatic stress [27].

Joinson [28] first used the term compassion fatigue to describe a nurse's loss of the ability to nurture in the setting of emergency departments. A sense of apathy and detachment in nurses was associated with multiple environmental stressors, complex patient needs, traumatic injury, and emotional distress [28]. Compassion fatigue is characterized by compassion discomfort, compassion stress, and finally fatigue, "where the compassion energy that is expended by nurses surpasses their ability to recover from this energy expenditure, resulting in significant negative psychological and physical consequences" [29]. The result of compassion fatigue is decreased sympathy and empathy, and an impaired ability to make decisions to care for patients [30].

Compassion is best defined by the Dali Lama as the wish that others should be free from suffering [31]. A thought-provoking definition describes compassion as a "full immersion into the condition of being human" [32]. Based on these definitions, it is not a surprise that compassion the part of a nurse may also lead to compassion fatigue. Compassion fatigue describes the negative feelings that nurses have when caring for those who are suffering. Compassion fatigue is brought about by relationships with others and, in turn, compassion fatigue on the part of the nurse affects her quality of life and the quality of life of those she is caring for [33].

Compassion fatigue is consistent with a reduced capacity in feeling empathy and a non-interest in bearing the suffering of others [34]. The natural outcomes of compassion fatigue are decreased empathetic responses to patients, withdrawal from care, and an imbalance of empathy and objectivity [29].

Literature relevant to studies about compassion and compassion fatigue and the way the concepts influence nurses' attitudes towards the suicidal patient were not found. However, one qualitative study describes the importance of compassionate care to the suicidal patient. Samuelsson, et al. [35] interviewed patients who had survived a suicide attempt $(n=18)$ to learn more about their experiences with caregivers during their hospitalization. In regard to perception of the caregivers and the care they received, there were both negative and positive statements.

On a positive note, one patient stated, "being in their care gave me a sense of security" and another stated "you feel welcome, relaxed, and secure" [35]. On a more negative note were these statements, "there was nobody who cared. Nobody took care of me, at least that's what I thought" and "he had emergency duty the night before so he was tired. It felt difficult, like being a burden. My being here is causing trouble" [35].

Samuelsson, et al. [35] concluded that positive and caring interactions between nurses and suicidal patients were crucial in the patient's desire to continue living. Health care personnel who are "devoted, hope-mediating, and committed" [35] are essential to providing therapeutic care to the suicidal patient.

\section{Discussion}

Nurses' attitudes towards the direct care of suicidal patients has not been studied extensively. However, this review of the literature supports the importance of the pursuit of knowledge related to the factors that influence nurses' attitudes toward caring for patients having suicidal thoughts and behaviors. Based on this integrative literature review, a number of factors related to nurses' attitudes emerged including the components of attitude, socio-demographic characteristics of the caregiver, the perceived professional capacity to care of the suicidal patient, and the relationship of compassion to attitude.

The specific components of attitude - cognitive, affective, and behavioral - have a distinct relationship to overall attitude towards the suicidal patient. What a nurse knows and believes (cognitive), what a nurse feels (affective), and how a nurse responds and acts (behavioral) are all important factors related to the care the suicidal patient receives. Ouzouni, et al. [16] promoted the need to quantify the components of attitude in order to better understand how this data affected care.

Studies examined in this literature review explored the relationship between demographic characteristics such as age, religion, gender, and education. Studies revealed more negative attitudes towards suicidal patients in older nurses as compared to their younger counterparts [11]. Female nurses showed more sympathy and tolerance to suicidal patients than did males [10]. The results of the religious background were mixed with one study showing those who identified as religious showed a more positive attitude [16] and another study showing that those nurses who attended church on a regular basis were condemnatory towards the care of the suicidal patient [20]. Finally, Sun, et al. [21] showed that there was a significant relationship between a nurses' level of education and her attitude towards the suicidal patient. Those nurses with higher levels of education showed a more positive attitude towards their patients with suicidal thoughts and behaviors [21].

Nurses must feel confident in their knowledge to assess, intervene, and care for the suicidal patient. An important aspect affecting attitude is the knowledge of the nurse in caring for the patient exhibiting suicidal thoughts and behaviors [24]. Suicide prevention education at the pre-licensure level and in professional continuing education varies across campuses and hospitals. Jacobson [24] demonstrated that increased knowledge and perceived professional capacity leads to improved attitudes towards suicide prevention. 
Compassion satisfaction and compassion fatigue are two important aspects of a nurses' professional quality of life [27]. The Emergency Nurses' Association (ENA) posits that compassion fatigue and burnout will lead to compromised decision-making ability and delayed reaction time with a direct impact on patient safety [36]. According to a metaanalysis by Sorenson [37] compassion fatigue leads to a decreased ability to feel empathy, avoidance of particular situations, and poor judgment. All of these symptoms lead to a diminished ability to interact therapeutically with patients [37] which is a critical problem when managing and caring for suicidal patients.

\section{Conclusion}

Attitudes towards the suicidal patient have an overall affect on suicidal thoughts, attempts, and behaviors [1]. Overall attitude is informed by the affective, behavioral, and cognitive components of attitude. Nurse demographics, especially age, gender, religion, and education - have a significant relationship to attitude towards the suicidal patient. The self-reported level of professional capacity to care for the suicidal patient has a direct correlation with nurses' attitude towards caring for those patients who are experiencing suicidal thoughts and behaviors. Although more research is needed to understand the relationship between compassion and attitude, the literature clearly shows that a nurse has a reduced ability to feel sympathy and empathy and an impaired ability to make decisions and to care for patients when experiencing compassion fatigue [30].

Research has shown that nurses have an overall negative attitude towards the care of the suicidal patient [14]. Opportunities for nurses to recognize and intervene therapeutically with suicidal patients may be thwarted by these negative attitudes [13]. In turn, this vulnerable patient population may sense negative attitudes from their nurses an miss opportunities to engage in therapeutic and healing relationships.

\section{Relevance to Nursing Practice}

Nurses can and will interact with patients who manifest suicide thoughts and behaviors across all hospital settings. Suicide is best conceptualized as a process rather than an end point emphasizing that nurses who care for the suicidal patient have the ability to provide positive therapeutic relationships and the ability to potentially intercede in life-saving measures. Opportunities for nurses to recognize and intervene therapeutically with suicidal patients may be thwarted by negative attitudes. Vulnerable patients may sense negative attitudes from their nurses and miss opportunities to engage and to heal.

This review highlights the need for all nurses in general hospital settings to be prepared for the care of the suicidal patient. A nurse's positive attitude towards a suicidal patient can be key in preventing a future suicide attempt or a completed suicide [14]. The establishment of continuing education that challenges mindsets, enhances knowledge, and improves a nurses' self-reported professional capacity to care for this vulnerable population may improve a nurses' attitude towards suicidal patients and may ultimately improve patient safety and patient outcomes [38].

\section{Disclosure}

The authors have confirmed that all authors meet the ICMJE criteria for authorship credit (www.icmje.org/ethical_lauthor.html), as follows: (1) substantial contributions to conception and design, acquisition of data, or analysis and interpretation of data, (2) drafting the article or revising it critically for important intellectual content, and (3) final approval of the version to be published.

\section{Conflict of Interest}

No conflict of interest has been declared by the authors.

\section{References}

1. Bolster C, Holliday C, Oneal G, Shaw M (2015) Suicide assessment and nurses: What does the evidence show? Online J Issues Nurs 20: 2.

2. World Health Organization (2012a) Public health action for the prevention of suicide: A framework. Geneva, Switzerland.

3. World Health Organization (2012b) Suicide data. Mental Health.

4. World Health Organization (2014) First WHO report on suicide prevention. Geneva, Switzerland.

5. Lipari R, Piscopo K, Kroutil L, Miller G (2015) Suicidal thoughts and behavior among adults: Results fomr the 2014 National Survey on Drug Use and Health. Substance Abuse and Mental Health Services Administration.

6. Owens PL, Fingar KR, Heslin K, Mutter R, Booth C (2017) Emergency department visits related to suicidal ideation, 2006-2013. Statistical Brief \#220, H-CUP.

7. Agency for Healthcare Research and Quality (2014) Overview of the Nationwide Emergency Department Sample (NES). HCUP.

8. Joint Commission (2016) Detecting and treating suicide ideation in all settings (Sentinel alert event) 56: 1-7.

9. U.S. Department of Health and Human Services (2012) 2012 National strategy for suicide prevention: Goals and objectives for action: A report of the U.S. Surgeon General and of the National Action Alliance for Suicide Prevention. Washington.

10. Ouzouni F, Eskin M (2006) Therapeutic and nontherapeutic reactions in a group of nurses and doctors in Turkey to patients who have attempted suicide. Social Behavior and Personality: An International Journal 34: 891-905.

11. Neville K, Roan N (2013) Suicide in hospitalized medical-surgical patients: Exploring nurses' attitudes. J Psychosoc Nurs Ment Health Serv 51: 35-43.

12. Mårtensson G, Jacobsson JW, Engström M (2014) Mental health nursing staff's attitudes towards mental illness: an analysis of related factors. J Psychiatr Ment Health Nurs 21: 782-788.

13. Joiner T (2009) The interpersonal-psychological theory of suicidal behavior: Current empirical status. Psychological Science Agenda. American Psychological Association.

14. Ouzouni C, Nakakis K (2013) Nurses' attitudes towards attempted suicide. Health Science Journal 7: 119-134.

15. Berlim MT, Perizzolo J, Lejderman F, Fleck MP, Joiner TE (2007) Does a brief training on suicide prevention among general hospital personnel impact their baseline attitudes towards suicidal behavior? J Affect Disord 100: 233-239.

16. Ouzouni C, Nakakis K (2009) Attitudes towards attempted suicide: the development of a measurement tool. Health Science Journal 3: 222-231.

17. Jain V (2014) 3D model of attitude. International Journal of Advanced Research in Management and Social Sciences 3: 1-12.

18. Wicker A (1969) Attitudes versus action: The relationship of verbal and overt behavioral responses to attitude objects. Journal of Social Issues 25: 41-78.

19. Domino G (1982) Suicide Opinion Questionnaire: Results of factor analytic studies Journal of Clinical Psychology 38: 257-262.

20. Botega NJ, Reginato D, Volk da Silva S, Filinto da Silva Cais C, Rapeli C, et al. (2005) Nursing personnel attitudes towards suicide: the development of a measure scale. Revista Brasileira de Psiquiatria.

21. Sun FK, Long A, Boore J (2007) The attitudes of casualty nurses in Taiwan to patients who have attempted suicide. J Clin Nurs 16: 255-263.

22. American Nurses Association (2014) Professional role competence. Professional standards, practise, ANA.

23. McAllister M, Creedy D, Moyle W, Farrugia C (2002) Nurses' attitudes towards clients who self-harm. $J$ Adv Nurs 40: 578-586.

24. Jacobson J, Osteen P, Jones A, Berman A (2012) Evaluation of the recognizing and responding to suicide risk training. Florida State University.

25. Sun FK, Long A, Huang XY, Chiang CY (2011) A quasi-experimental investigation into the efficacy of a suicide education programme for second-year student nurses in taiwan. J Clin Nurs 20: 837-846. 
26. Stamm BH (2010) The ProQoL Concise Manual.

27. Stamm B (2016) Professional quality of life elements theory and measurement: Compassion satisfaction and compassion fatigue, burnout, secondary traumatic stress, vicarious tramatization and vicarious transformation.

28. Joinson C (1992) Coping with compassion fatigue. Nursing 22: 116-121.

29. Boyle DA (2011) Countering Compassion Fatigue: A Requisite Nursing Agenda. Online J Issues Nurs 16: 2.

30. Cocker F, Joss N (2016) Compassion Fatigue among Healthcare, Emergency and Community Service Workers: A Systematic Review. Int J Environ Res Public Health 13: pii: E618.

31. Lama D (2002) An open heart: Practicing compassion in everyday life. Vreeland N (Ed) Boston: Back Bay Books.

32. McNeill DP, Morrison D, Nouwen H (1982) Compassion: A reflection on the Christian life. New York.
33. Todaro-Franceschi V (2013) Compassion fatigue and burnout in nursing: Enhancing professional quality of life. Springer Publishing Company, New York.

34. Adams R, Boscarino J, Figley C (2009) Compassion fatigue and psychological distress among social workers: A validation study. Am J Orthopsychiatry 76:103-108.

35. Samuelsson M, Wiklander M, Asberg M, Saveman B (2000) Psychiatric care as seen by the attempted suicide patient. J Adv Nurs 32: 635-643.

36. Walsh R (2013) Nurse fatigue. Emergency Nurses' Association, ENA

37. Sorenson C, Bolick B, Wright K, Hamilton R (2016) Understanding Compassion Fatigue in Healthcare Providers: A Review of Current Literature. J Nurs Scholarsh 48: $456-465$

38. Anderson M, Standen J (2007) Attitudes towards suicide among nurses and doctors working with children and young people who self-harm. J Psychiatr Ment Health Nurs 14: $470-477$.

Copyright: (C2018 Stevens KP. This is an open-access article distributed under the terms of the Creative Commons Attribution License, which permits unrestricted use, distribution, and reproduction in any medium, provided the original author and source are credited. 